\title{
Comparison of Occurrence of Bone Tunnel Laceration, Clinical Results, and Cuff Repair Integrity of Transosseous Arthroscopic Rotator Cuff Repair With and Without Lateral Cortical Augmentation
}

\author{
Yoshihiro Hirakawa, M.D., Tomoya Manaka, M.D., Yoichi Ito, M.D., \\ Yoshinobu Matsuda, M.D., Katsumasa Nakazawa, M.D., Ryosuke Iio, M.D., and \\ Hiroaki Nakamura, M.D.
}

\begin{abstract}
Purpose: To compare the occurrence of bone tunnel laceration, the short-term clinical results, and cuff repair integrity of transosseous arthroscopic rotator cuff repair (ARCR) using a tunneling device, with and without lateral cortical augmentation. Methods: A retrospective review of patients who underwent transosseous ARCR from May 2012 to December 2017 was conducted. The inclusion criterion was repairable medium- to massive-sized full-thickness rotator cuff tear. This study included 2 consecutive series of patients undergoing transosseous ARCR with and without lateral cortical augmentation, called the ITO method and AT method, respectively. The incidence of bone tunnel laceration was evaluated intraoperatively. Patients were assessed through a range of motion and Constant scores preoperatively and at final follow-up. Further, magnetic resonance imaging was performed at 24 months postoperatively to examine the repaired rotator cuff integrity. Results: A total of 121 subjects were included: 33 in the AT group and 88 in the ITO group. The intraoperative bone tunnel laceration occurrence rate was $67 \%$ and $4 \%$ for the AT and ITO methods, respectively; the difference was significant $(P=.001)$. Anatomic failure rate (Sugaya III, IV, and V) rate for medium- to large-sized tears was significantly lower for the ITO than for the AT method $(29 \%$ vs $65 \%, P=.004)$, but not for massive tears $(61 \%$ vs $69 \%, P=.515)$. The mean forward elevation, abduction, external rotation, internal rotation, and Constant score were significantly improved at final follow-up from preoperative values. There were no significant differences between the 2 methods. Conclusions: Transosseous ARCR using a tunneling device with and without lateral cortical augmentation is a reliable method of improving clinical results at a minimum follow-up of 2 years. The intraoperative occurrence rate of bone tunnel laceration occurrence rate and the anatomic failure rate of medium- to large-sized cuff tear were lower with lateral cortical augmentation than without it. Level of Evidence: Level IV, therapeutic cases series.
\end{abstract}

\section{Introduction}

$\mathbf{S}^{\mathrm{e}}$ everal methods for performing arthroscopic rotator cuff repair (ARCR) exist. ARCR using suture anchors has become the most common procedure, and excellent clinical results have been reported. ${ }^{1-4}$ However, using suture anchors has certain disadvantages, such as dislocation, ${ }^{5}$ cost $^{6,7}$ osteolysis, ${ }^{8,9}$ problems with revision surgery, ${ }^{10}$ and perianchor reactions. ${ }^{11,12}$

From the Department of Orthopaedic Surgery, Osaka City University Graduate School of Medicine (Y.H., T.M., K.N., R.I., H.N.); Ito Clinic, Osaka Shoulder Center (Y.I.); and Nagayoshi General Hospital (Y.M.), Osaka, Japan.

The authors report that they have no conflicts of interest in the authorship and publication of this article. Full ICMJE author disclosure forms are available for this article online, as supplementary material.

Received July 8, 2020; accepted February 10, 2021.

Address correspondence to Tomoya Manaka, M.D., Ph.D., Department of Orthopaedic Surgery, Osaka City University Graduate School of Medicine, 1-4-3,
To avoid these issues, some surgeons choose transosseous ARCR techniques instead. ${ }^{13-18}$ There are various approaches to transosseous ARCR, but these are generally all difficult to perform. However, with the development of a tunneling device, implementing transosseous ARCR has become easier, and we commenced the use of this procedure in our institute in May 2012.

\footnotetext{
Asahimachi, Abeno-ku, Osaka, 545-8585 Japan. E-mail: manaka@med. osaka-cu.ac.jp

(C) 2022 THE AUTHORS. Published by Elsevier Inc. on behalf of the Arthroscopy Association of North America. This is an open access article under the CC BY-NC-ND license (http://creativecommons.org/licenses/by-nc-nd/4.0/). 2666-061X/201193

https://doi.org/10.1016/j.asmr.2021.02.004
} 
A previous study reported that clinical results following transosseous ARCR using a tunneling device without lateral cortical augmentation are significantly improved at a minimum 2-year follow-up. However, intraoperative bone tunnel laceration was observed in $67.8 \%$ of all tunnels, and this study revealed that bone tunnel laceration affects rotator cuff repair integrity. ${ }^{19}$ Other reports of transosseous ARCR using a tunneling device also stated that this procedure frequently is accompanied by bone tunnel laceration during knottying. ${ }^{20,21}$ In addition, one paper using magnetic resonance imaging (MRI) demonstrated that more than $80 \%$ of the bone tunnel morphology had changed at 6 months postoperatively. ${ }^{19}$ The original shape of the bone tunnel made at the operation time should be maintained to avoid damaging the cancellous bone. To preserve the original L-shaped bone tunnel morphology and prevent bone tunnel laceration, in May 2013 lateral cortical augmentation was introduced in a technique named the interposed transosseous (ITO) method.

Several studies on transosseous ARCR using a tunneling device with lateral cortical augmentation have been reported. ${ }^{22,23}$ However, little is known about the influence of lateral cortical augmentation on the clinical results and cuff repair integrity after performing transosseous ARCR using a tunneling device. The purpose of this study was to compare occurrence of bone tunnel laceration, the short-term clinical results, and cuff repair integrity of transosseous ARCR using a tunneling device, with and without lateral cortical augmentation. We hypothesized that lateral cortical augmentation would reduce the occurrence of bone tunnel laceration and affect both the clinical results and cuff repair integrity.

\section{Methods}

\section{Patient Selection}

This study was retrospective and included consecutive patients who underwent transosseous arthroscopic rotator cuff repair. Between May 2012 and April 2013, patients $(\mathrm{n}=33)$ who underwent surgical repair of a rotator cuff tear using a tunneling device without lateral cortical augmentation (AT method), and between May 2013 and December 2017, patients $(n=88)$ who underwent surgery for the same repair using a tunneling device, but with lateral cortical augmentation (ITO method), were included in this study. We retrospectively followed up with the patients. The inclusion criterion was repairable medium- to massive-sized full-thickness rotator cuff tear. Exclusion criteria were as follows: (1) revision rotator cuff repair; (2) transosseous ARCR with additional anchors or sutures; (3) partial rotator cuff repair; and (4) no additional procedure such as capsulotomy or acromial clavicular resection. This study was approved by our institutional review board (Elucidation of the pathological condition of the shoulder disease after having undergone arthroscopic surgeries [retrospective multicenter clinical study], protocol identification number: 3221 ). All patients agreed to participate in the study.

\section{Surgical Technique}

This procedure was completed by 2 different surgeons (Y.I. and T.M.). Shoulder arthroscopy was achieved while the patient was under general anesthesia in the lateral decubitus position. The upper limb was maintained at the abduction of approximately $60^{\circ}$.

The glenohumeral arthroscopic inspection was performed, and the size and proportions of the rotator cuff tear and condition of the long head of the biceps were assessed. In cases of tendinopathy of the long head of the biceps, a tenotomy was implemented. Arthroscopic subacromial decompression was performed in all cases. After the rotator cuff repair was prepared for the procedure, a drill hole was made and a tunneling device (i.e., the ARTHROTUNNELER; Wright Medical, Memphis, TN) (Fig 1A) was placed in the drill hole. This device formed an angled bone tunnel, with the length of the lateral tunnel being $15 \mathrm{~mm}$. A shuttle suture was introduced through the device via loop retrieval (Fig 1B), which was then replaced by 3 braided nonabsorbable sutures (FiberWire; Arthrex, FL) in each drill hole. For the ITO method, these sutures were used to install an ALLthread Knotless Suture Anchor (Biomet, Warsaw, IN) on the lateral side of the greater tuberosity to act as the lateral cortical augmentation (Fig $1 \mathrm{C}$ and D). The length of this augmentation was the same as that of the lateral tunnel (Fig 2). For the AT method, no lateral cortical augmentation was established at the greater tuberosity. The appropriate number of holes was made depending on the cuff tear size.

Passive range-of-motion exercises were started from the day after the operation. The abduction sling was removed, and active-assisted range-of-motion exercises were initiated at 6 weeks postoperatively.

\section{Evaluation}

The shoulder range of motion and Constant score ${ }^{24}$ were assessed preoperatively and postoperatively at the final follow-up. Four physical therapists conducted these evaluations. The rotator cuff tear size and presence of bone tunnel laceration after knot tying were evaluated intraoperatively. For bone tunnel laceration, a positive result was defined as a cutout of greater than $5 \mathrm{~mm}$ for the AT method (Fig 3A), and movement from the original position to another position for the ITO method (Fig 3B). All patients who underwent ARCR at the authors' institution were advised to undergo an MRI evaluation of cuff repair conditions 24 months postoperatively, and an MRI was used to evaluate the Sugaya classification. ${ }^{25}$ This study defined complete retear as Sugaya type IV and V and anatomic repair 
Fig 1. Arthroscopic surgery findings (left side): (A) a tunneling device was introduced into the medial portal; (B) shuttle suture was introduced through the device via loop retrieval; (C) lateral cortical augmentation was introduced into the greater tuberosity via the suture using anterolateral portal; and (D) arthroscopic findings after the completed repair from anterolateral portal.

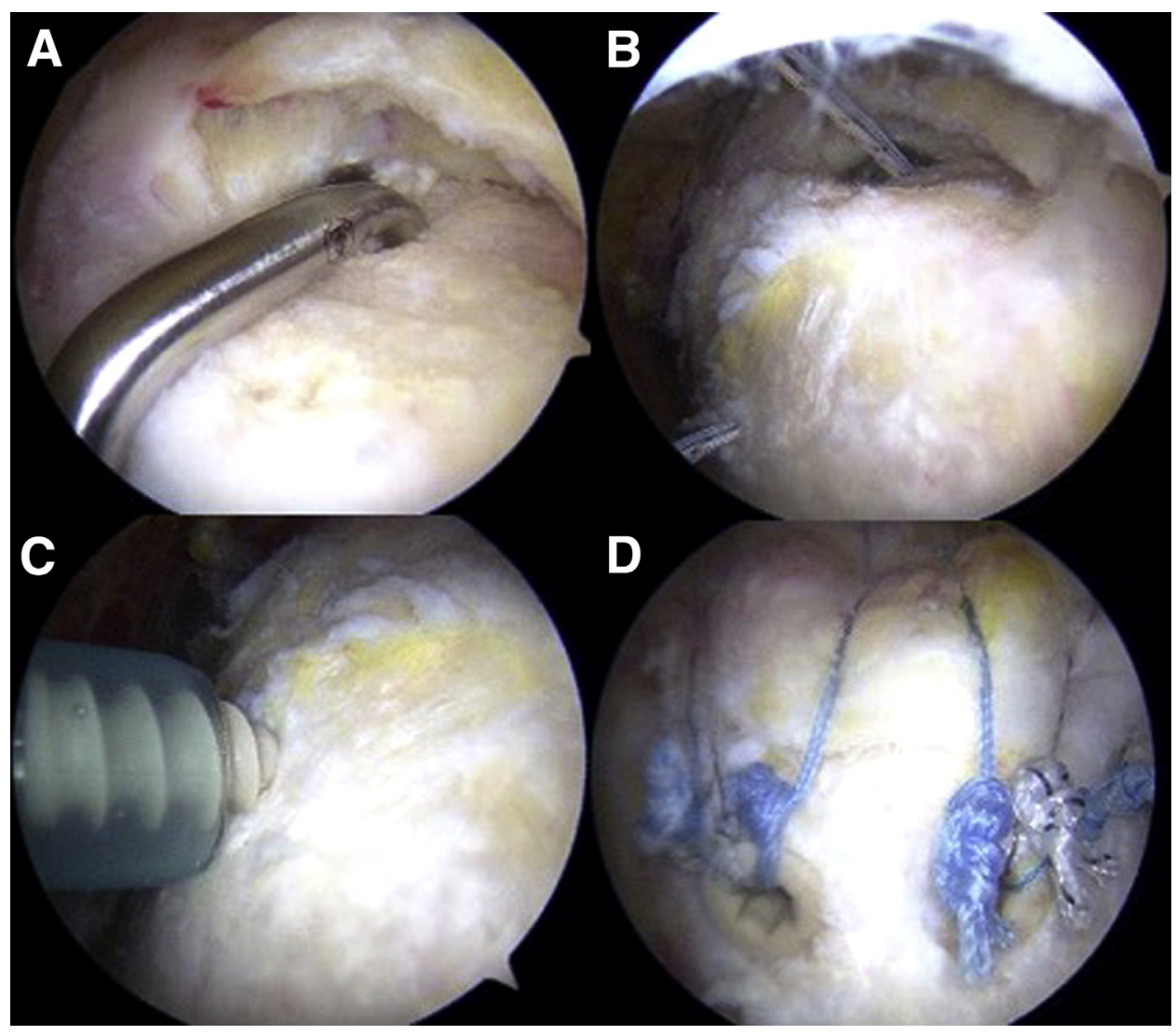

failure $^{21}$ as Sugaya type III, IV, and V. A shoulder surgeon (Y.H.) interpreted the MRI findings and determined the Sugaya classification.

\section{Statistical Analysis}

Statistical analyses were performed using GraphPad prism statistical software, version 8 (GraphPad Software,
San Diego, CA). The paired $t$ test, Fisher exact test, and the Mann-Whitney $U$ test were used to compare variables in this study. A $P$ value of $<.05$ was considered to be statistically significant.

We made a post hoc power analysis on our results with a specific statistical software (G*Power, version 3.1.2, Heinrich Heine University, Dusseldorf, Germany).

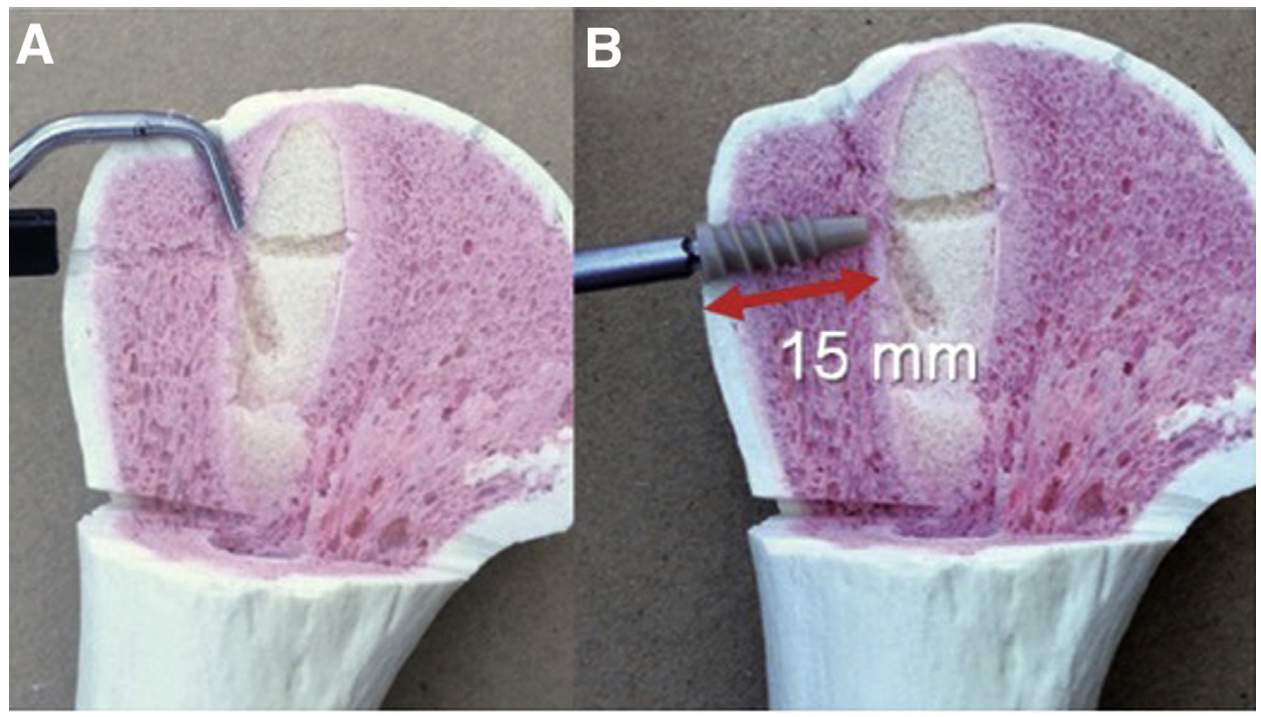

Fig 2. Bone model of the humerus. (A) Vertical shape of the bone tunnel; and (B) lateral cortical augmentation was the same length as that of the bottom side. 


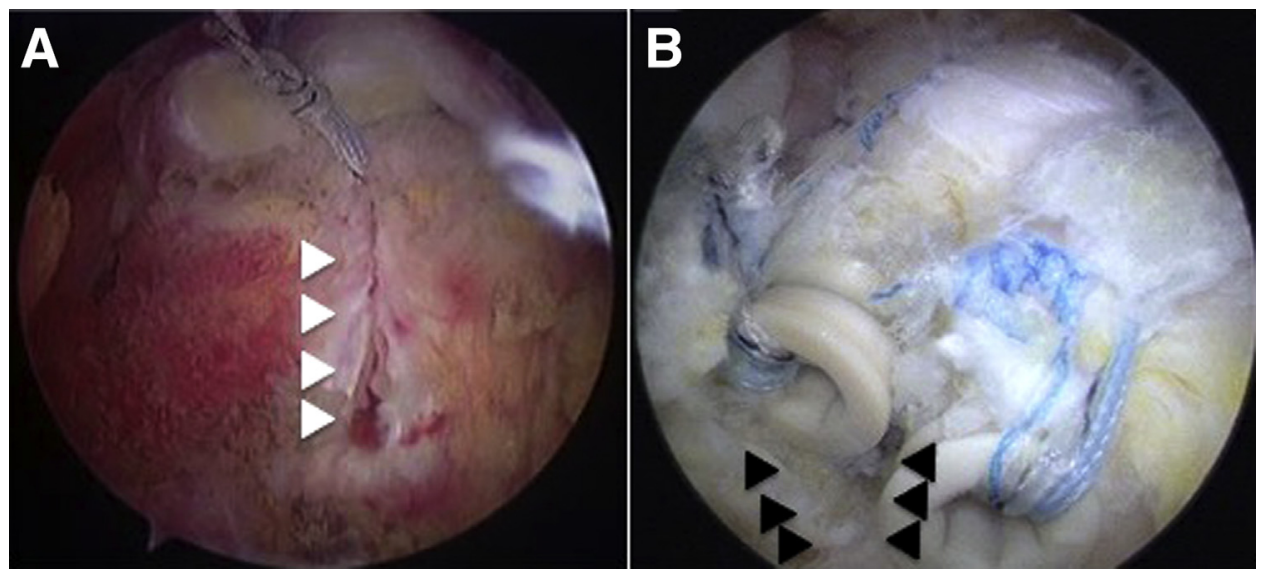

Fig 3. (A) Bone tunnel laceration of the greater tuberosity from the AT method (white arrows); and (B) Bone tunnel laceration of the greater tuberosity from the ITO method (black arrows). (AT, transosseous arthroscopic rotator cuff repair [ARCR] using a tunneling device without lateral cortical augmentation; ITO, interposed transosseous ARCR/transosseous ARCR using a tunneling device with lateral cortical augmentation)

According to the post hoc power analysis, our study power to detect a significant difference is 0.80 with and an alpha error of 0.05 .

\section{Results}

\section{Patient Data}

Of 186 consecutive patients who underwent surgical repair of a rotator cuff tear using a tunneling device, 39 patients were excluded from this study. This study enrolled 147 patients, who were divided into 2 groups: the AT method ( 39 patients) and the ITO method (108 patients). Of these, 26 patients dropped out of the study: 9 patients ( 3 patients: AT, 6 patients ITO) dropped out because of a need for revision surgery within 2 years after primary surgery, and 17 patients ( 3 patients: AT, 14 patients ITO) dropped out postoperatively. A total of 121 subjects were ultimately included: 33 in the AT group and 88 in the ITO group. The follow-up rate was $82 \%$ $(121 / 147)$. A flow diagram illustrates the grouping and flow of patients in our clinical study (Fig 4). The demographic characteristics, including age, sex, mean follow-up period, number of biceps tenotomy, and size of the rotator cuff tear, are shown in Table 1.

\section{Clinical Outcome}

Range of motion and constant score were evaluated at the last follow-up visits showed significant improvement compared with preoperative scores in both methods, except for the external rotation of the AT
Fig 4. Flow diagram to show the grouping of the patients included in the study. (AT, transosseous arthroscopic rotator cuff repair [ARCR] using a tunneling device without lateral cortical augmentation; ITO, interposed transosseous ARCR/transosseous ARCR using a tunneling device with lateral cortical augmentation)

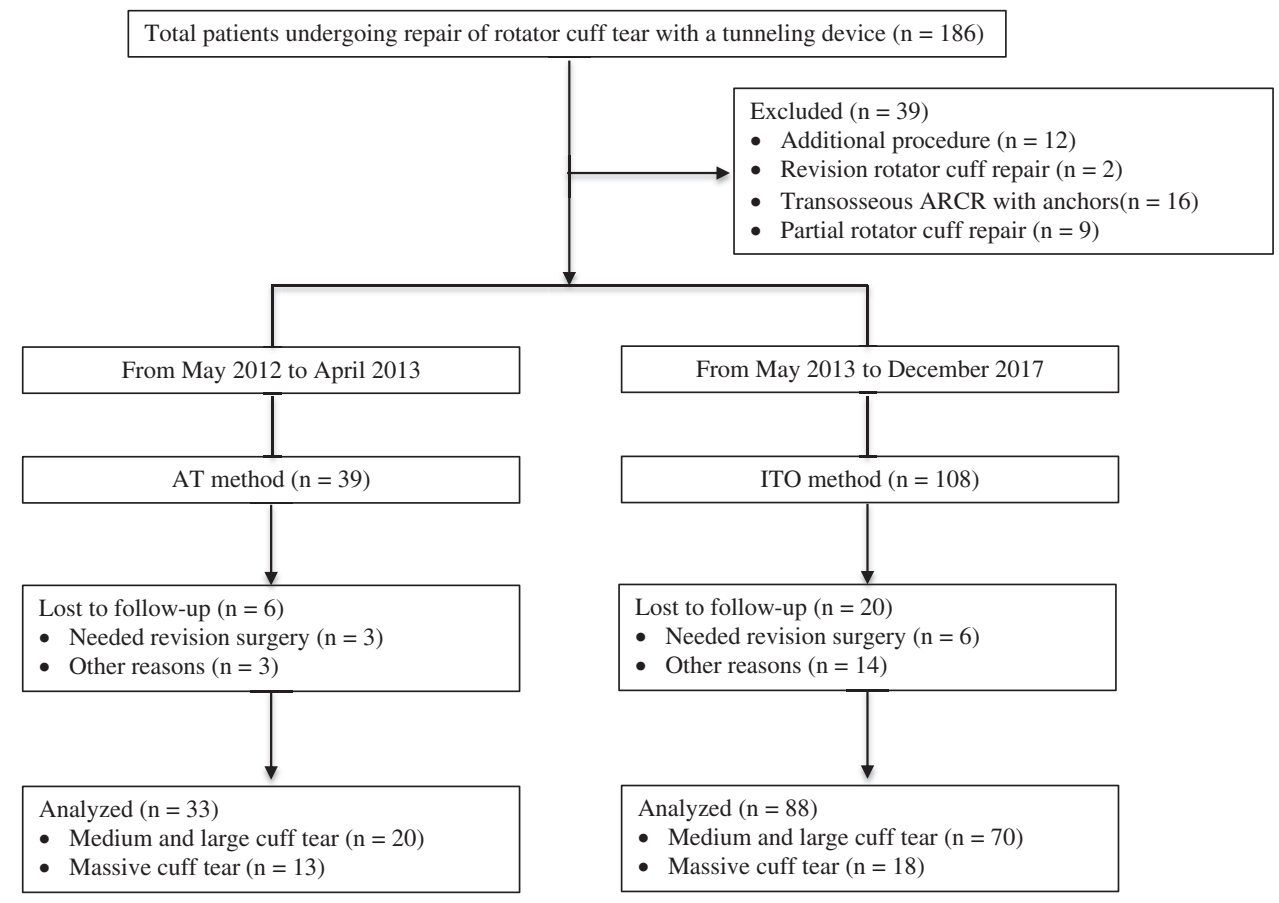


Table 1. Demographic and Surgical Data

\begin{tabular}{|c|c|c|c|}
\hline & At Method & ITO Method & $P$ Value \\
\hline Number & 33 & 88 & \\
\hline $\begin{array}{l}\text { Age, } y, \text { mean } \pm S D \\
\quad(\text { range) }\end{array}$ & $68.6 \pm 8.7(51-83)$ & $65.0 \pm 9.3(48-85)$ & $.061^{*}$ \\
\hline Sex, male/female, $\mathrm{n}$ & $21: 12$ & $47: 41$ & $.337 \dagger$ \\
\hline $\begin{array}{l}\text { Follow up, mo, } \\
\text { mean } \pm \text { SD } \\
\text { (range) }\end{array}$ & $30.5 \pm 4.1(24-39)$ & $27.1 \pm 5.9(24-48)$ & $.001^{*}$ \\
\hline \multicolumn{4}{|l|}{ Cuff tear size } \\
\hline $\begin{array}{l}\text { Medium- and } \\
\text { large-sized tear }\end{array}$ & 20 & 70 & \\
\hline Massive-sized tear & 13 & 18 & $.060 \dagger$ \\
\hline \multicolumn{4}{|c|}{$\begin{array}{l}\text { ARCR, arthroscopic rotator cuff repair; AT, transosseous ARCR } \\
\text { using a tunneling device without lateral cortical augmentation; ITO, } \\
\text { interposed transosseous ARCR/transosseous ARCR using a tunneling } \\
\text { device with lateral cortical augmentation; SD, standard deviation. } \\
{ }^{*} \text { Mann-Whitney } U \text { test. } \\
{ }^{\dagger} \text { Fisher exact test. }\end{array}$} \\
\hline
\end{tabular}

method. However, no significant differences were found in range of motion (forward elevation, $P=.392$; abduction, $P=.108$; external rotation, $P=.100$; internal rotation, $P=.303)$ and Constant score $(P=.354)$ between the 2 methods. Both methods demonstrated significant improvement of Constant score according to the minimal clinically important difference criteria for Constant score $(10.4)^{26}$ (Table 2).

\section{Bone Tunnel and Bone Tunnel Laceration}

The number of bone tunnels was significantly greater in the AT method than in the ITO method. However, the occurrence rate of bone tunnel laceration was significantly greater in the AT method than in the ITO method (Table 3).

\section{Cuff Repair Integrity}

No significant difference of retear rate between the AT method and ITO method were observed in mediumto large-sized and massive-sized tear. However, the anatomic failure rate of medium to-large-sized rotator cuff repair in the ITO method was significantly lower than that in the AT method $(P=.004)$. However, there was no significant difference in the anatomic failure rate of massive rotator cuff repair between the AT method and ITO method $(P=.515)$ (Table 4$)$.

\section{Discussion}

The current study results showed that transosseous ARCR using a tunneling device was associated with a positive range of motion and clinical outcomes at a minimum follow-up of 2 years, with or without lateral cortical augmentation. Furthermore, lateral cortical augmentation could reduce the occurrence of bone tunnel lacerations. However, a significant difference was found between the AT and ITO methods in the anatomic failure rate of medium- to large-sized rotator cuff tears. Therefore, our hypothesis of lateral cortical augmentation affecting bone tunnel laceration and cuff repair healing for medium- to large-sized tears was proven, but not the clinical outcomes.

Several clinical reports on transosseous ARCR using a tunneling device, same as this study, have been published. For example, Randelli et al. ${ }^{27}$ determined that the results of performing transosseous ARCR using a tunneling device are comparable with single-row repair using an anchor, at a minimum follow-up period of 15 months. However, they included no measures to assess bone tunnel laceration and did not report on the occurrence of this phenomenon. Black et al. ${ }^{20}$ observed positive clinical results in 31 patients following transosseous ARCR using a tunneling device. These authors also mentioned the incidence of intraoperative bone tunnel laceration during knot tying, and used cortical augmentation for $23 \%$ of all patients. Nevertheless, cortical augmentation was not significantly associated with improved outcome scores. Liu et al. ${ }^{21}$ described the favorable functional and anatomical outcomes

Table 2. Comparison Between Preoperative and Postoperative Range of Motion and Clinical Outcomes

\begin{tabular}{|c|c|c|c|c|c|c|c|}
\hline & \multicolumn{2}{|c|}{ At Method } & \multirow[b]{2}{*}{$P$ Value } & \multicolumn{2}{|c|}{ ITO Method } & \multirow[b]{2}{*}{$P$ Value } & \multirow{2}{*}{$\begin{array}{c}\text { At vs ITO at } \\
\text { Final Follow-Up } \\
P \text { Value }\end{array}$} \\
\hline & Preoperative & $\begin{array}{c}\text { Final } \\
\text { Follow-Up }\end{array}$ & & Preoperative & $\begin{array}{c}\text { Final } \\
\text { Follow-Up }\end{array}$ & & \\
\hline \multicolumn{8}{|l|}{ Shoulder ROM, mean \pm SD } \\
\hline Forward elevation, & $123 \pm 39$ & $152 \pm 24$ & $.032 *$ & $124 \pm 39$ & $158 \pm 18$ & $.001^{*}$ & $.392^{\dagger}$ \\
\hline Abduction, & $119 \pm 52$ & $148 \pm 28$ & $.002 *$ & $114 \pm 47$ & $156 \pm 24$ & $.001^{*}$ & $.108^{\dagger}$ \\
\hline External rotation, & $36 \pm 27$ & $48 \pm 15$ & $.027^{*}$ & $40 \pm 19$ & $41 \pm 20$ & $.653 *$ & $.100^{\dagger}$ \\
\hline
\end{tabular}

NOTE. Data are reported as mean \pm SD.

ARCR, arthroscopic rotator cuff repair; AT, transosseous ARCR using a tunneling device without lateral cortical augmentation; ITO, interposed transosseous ARCR/transosseous ARCR using a tunneling device with lateral cortical augmentation; ROM, range of motion; SD, standard deviation.

*Paired $t$ test.

${ }^{\dagger}$ Mann-Whitney $U$ test. 
Table 3. Comparison of the Average Number of Tunnels and Occurrence Rate of Bone Tunnel Laceration

\begin{tabular}{lccc}
\hline & At Method & ITO Method & $P$ Value \\
\hline $\begin{array}{l}\text { Number of tunnels, median } \\
\text { (range) }\end{array}$ & & & \\
Medium- and large-sized tear & $2(1-2)$ & $1(1-2)$ & $.001^{*}$ \\
$\quad$ & $3(2-4)$ & $2(1-3)$ & $.001^{*}$ \\
Massive-sized tear & $2(1-4)$ & $2(1-3)$ & $.001^{*}$ \\
$\begin{array}{l}\text { Total } \\
\text { Occurrence rate of bone tunnel }\end{array}$ & & & \\
$\quad$ laceration & & & \\
Medium- and large-sized tear & $64 \%(25 / 39)$ & $2 \%(2 / 98)$ & $.001^{*}$ \\
Massive-sized tear & $69 \%(25 / 36)$ & $8 \%(3 / 37)$ & $.001^{*}$ \\
Total & $67 \%(50 / 75)$ & $4 \%(5 / 135)$ & $.001^{*}$ \\
\hline
\end{tabular}

ARCR, arthroscopic rotator cuff repair; AT, transosseous ARCR using a tunneling device without lateral cortical augmentation; ITO, interposed transosseous ARCR/transosseous ARCR using a tunneling device with lateral cortical augmentation.

*Mann-Whitney $U$ test.

achieved at a minimum follow-up period of 2 years following transosseous ARCR using a tunneling device for symptomatic 2 - to 4 -cm full-thickness tears. In that study, $44 \%$ of all patients developed intraoperative bone tunnel laceration during the operation. There were no significant differences between the clinical outcomes of patients with and without bone tunnel laceration. However, they recommended the use of a cortical augmentation to minimize the risk of suture cut-through, concerning bone tunnel quality at the great tuberosity. Similar to previous reports, the current study revealed that the range of motion and clinical outcomes improved after transosseous ARCR using a tunneling device at a minimum 2-year follow-up, regardless of lateral cortical augmentation.

This study also observed that the number of bone tunnels created and the occurrence rate of bone tunnel laceration was greater for the AT method than the ITO method. In general, during knot-tying, bone tunnel lacerations form from the lateral edge of the bone tunnel. ${ }^{20,21}$ It can be considered that using 3 threads to 1 bone tunnel simultaneously in AT the method, thread stress was concentrated, and bone tunnel laceration was likely to occur. Moreover, if the laceration occurred at the first suture, the other 2 sutures could not be tightened firmly. In such cases, the creation of additional tunnels was required to recover the fixation force. Caldwell et al. ${ }^{28}$ reported that lateral cortical augmentation might increase the ultimate pull-out strength of sutures from the bone by 2 -fold. Similarly, other authors have also recommended the use of lateral cortical augmentation to enhance pull-out strength. ${ }^{29,30}$ Thus, lateral cortical augmentation should be used to improve suture pull-out strength and to prevent bone tunnel laceration in all cases.

No significant difference in the re-tear rate between the AT and ITO methods was shown. Randelli et al. ${ }^{27}$ and Black et al. ${ }^{20}$ reported a retear rate of $13.3 \%(4 / 30)$ and $9.7 \%$ (3/31), respectively, following transosseous ARCR using a tunneling device. These results agree with those of our study.

In contrast, the anatomic failure rate of medium- to large-sized rotator cuff tears for the AT method was significantly greater than for the ITO method. Furthermore, the AT method demonstrated a greater proportion of Sugaya type III retears than the ITO method. Salata et al. $^{31}$ described a biomechanical cadaveric study measuring the extent of initial gapping between the insertion site and the repaired tendon when using a tunneling device to repair a supraspinatus rotator cuff tear. According to this research, the gapping is $3 \mathrm{~mm}$ larger for a tunneling device than when using anchor fixation. A previous study revealed that in $90.1 \%(73 / 81)$ of patients who underwent surgery using the AT method, bone tunnel morphology changed from the original L-shaped to curve-shaped or straight-shaped according to an MRI at 6 months after the operation. ${ }^{19}$ The curveshaped and straight-shaped bone tunnel morphology was considered to indicate loosened suture strings after surgery. This might be due to a reduction in the thread

Table 4. Comparison of the Retear Rate and Anatomic Failure Rate

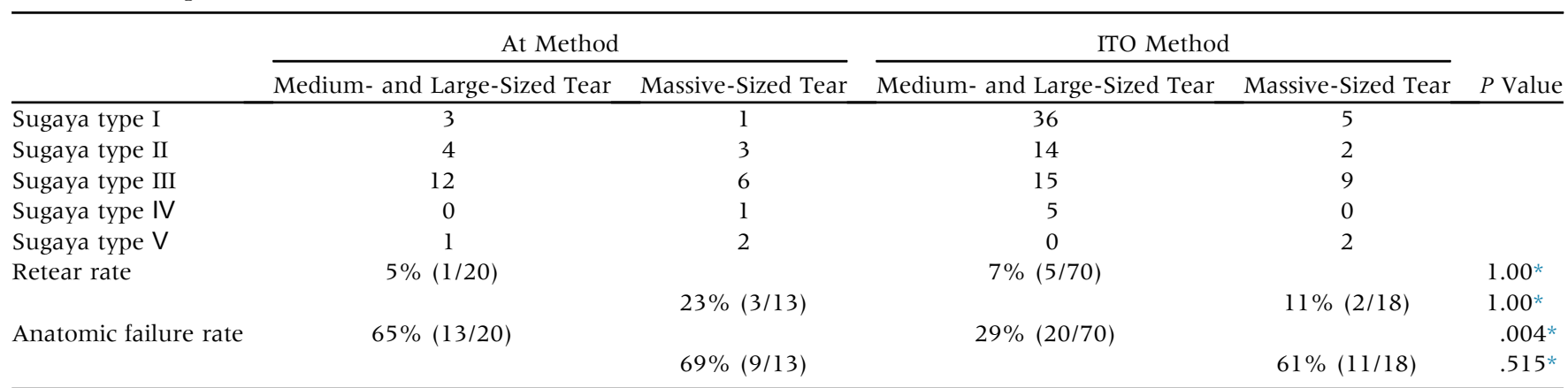

ARCR, arthroscopic rotator cuff repair; AT, transosseous ARCR using a tunneling device without lateral cortical augmentation; ITO, interposed transosseous ARCR/transosseous ARCR using a tunneling device with lateral cortical augmentation, retear (Sugaya IV and V), anatomic repair failure (Sugaya III, IV, and V).

*Fisher exact test. 
tension of the repaired cuff used for the AT method. This phenomenon may have caused partial retear (Sugaya type III). In contrast, curve-shaped or straight-shaped bone tunnel morphology was not recognized in patients following the ITO method at postoperatively MRI. Lateral cortical augmentation in the ITO method prevented the change of bone tunnel morphology.

However, no significant difference in anatomic failure rate for massive rotator cuff tears between the 2 methods was revealed. There are 2 possible reasons. First, because of the massive size of the tears, the condition of the rotator cuff was so poor that the cuff was torn by the suture thread regardless of any loosening. Galatz et al. ${ }^{32}$ reported that recurrent tears were seen in 17 of the 18 patients with massive rotator cuff tears. They determined this to be due to the poor-quality of the tissue. Furthermore, Choi et al. ${ }^{33}$ reported that the retear rate was statistically correlated with preoperative fatty generation and tear size only. Second, the thickness of the repaired rotator cuff was insufficient from the beginning because of inferior cuff quality, and thus it is possible that the cuff repair integrity classification was Sugaya type III immediately after surgery. Sugaya et al. ${ }^{25}$ reported that the proportion of type III repairs for large-to-massive cuff tears is greater than that for small-to-medium cuff tears. Therefore, incomplete healing after massive rotator cuff repair is not thought to be uncommon. As a result, for massive rotator cuff tears, lateral cortical augmentation may be not effective in aiding cuff repair healing. In general, massive rotator cuff tears are considered difficult to repair, and no consensus treatment has yet been reached. ${ }^{34-36}$ In the future, it is necessary to consider other procedures to achieve better results for massive rotator cuff tears.

Our study suggests that transosseous ARCR using a tunneling device with lateral cortical augmentation prevents intraoperative bone tunnel laceration. This procedure enhanced the cuff repair integrity of medium- to large-sized tears but did not affect the clinical outcomes at a minimum follow-up period of 2 years.

\section{Limitations}

The current study had several limitations. First, this study was a consecutive case series and could not completely eliminate the possibility of influencing the learning curve about the difference in bone tunnel laceration occurrence between 2 procedures. Second, the number of patients who underwent surgery using the AT method was relatively small compared with the ITO method. However, the post hoc analysis was conducted, and the anatomic failure rate difference has enough power.

\section{Conclusions}

Transosseous ARCR using a tunneling device with and without lateral cortical augmentation is a reliable method of improving clinical results at a minimum follow-up of 2 years. The intraoperative occurrence rate of bone tunnel laceration and the anatomic failure rate of medium- to large-sized cuff tear were lower with lateral cortical augmentation than without it.

\section{Acknowledgments}

We thank physical therapists Ayako Ogura, Teruya Yukawa, Yuki Uetani, and Sadamu Kofuji for patient evaluation of functional outcomes and range of motion.

\section{References}

1. Manaka T, Ito Y, Matsumoto I, Takaoka K, Nakamura H. Functional recovery period after arthroscopic rotator cuff repair: Is it predictable before surgery? Clin Orthop Relat Res 2011;469:1660-1666.

2. Sheean AJ, Hartzler RU, Burkhart SS. Arthroscopic rotator cuff repair in 2019: Linked, double row repair for achieving higher healing rates and optimal clinical outcomes. Arthroscopy 2019;35:2749-2755.

3. Kim JH, Kim YS, Park I, et al. A comparison of openconstruct PEEK suture anchor and non-vented biocomposite suture anchor in arthroscopic rotator cuff repair: A prospective randomized clinical trial. Arthroscopy 2020;36:389-396.

4. Lee KW, Yang DS, Lee GS, Ma CH, Choy WS. Clinical outcomes and repair integrity after arthroscopic fullthickness rotator cuff repair: suture-bridge versus double-row modified Mason-Allen technique. J Shoulder Elbow Surg 2018;27:1953-1959.

5. Vonhoegen J, John D, Hagermann C. Osteoconductive resorption characteristics of a novel biocomposite suture anchor material in rotator cuff repair. J Orthop Surg Res 2019;14:12.

6. Black EM, Austin LS, Narzikul A, Seidl AJ, Martens K, Lazarus MD. Comparison of implant cost and surgical time in arthroscopic transosseous and transosseous equivalent rotator cuff repair. I Shoulder Elbow Surg 2016;25:1449-1486.

7. Narvy SJ, Ahluwalia A, Vangsness CT Jr. Analysis of direct costs of outpatient arthroscopic rotator cuff repair. Am J Orthop 2016;45:E7-E11.

8. Stahnke K, Nikulka C, Diederichs G, Haneveld H, Scheibel M, Gerhardt C. Serial MRI evaluation following arthroscopic rotator cuff repair in double-row technique. Arch Orthop Trauma Surg 2016;136:665-672.

9. Micic I, Kholinne E, Kwak JM, Koh KH, Jeon IH. Osteolysis is observed around both bioabsorbable and nonabsorbable anchors on serial magnetic resonance images of patients undergoing arthroscopic rotator cuff repair. Arch Orthop Trauma Surg 2019;53:414-419.

10. Reda B, Coady C, Wong I. Revision of failed rotator cuff reconstruction with a large humeral head cyst. Arthrosc Tech 2017; 5:e2023-e2030.

11. Kim SH, Yang SH, Rhee SM, Lee KJ, Kim HS, Oh JH. The formation of perianchor fluid associated with various suture anchors used in rotator cuff repair. Bone Joint $J$ 2019;101-B:1506-1511. 
12. Ro K, Pancholi S, Son HS, Rhee YG. Perianchor cyst formation after arthroscopic rotator cuff repair using allsuture-type, bioabsorbable-type, and peek-type anchors. Arthroscopy 2019;35:2284-2292.

13. Plachel F, Traweger A, Vasvary I, Schanda JE, Resch H, Moroder P. Long-term results after arthroscopic transosseous rotator cuff repair. J Shoulder Elbow Surg 2019;28: 706-714.

14. Chillemi C, Mantovani M. Arthroscopic trans-osseous rotator cuff repair. Muscles Ligaments Tendons J 2017;7: 19-25.

15. Murphy MM, Tashjian RZ, Burks RT. Rotator cuff repair technique with transosseous knotless anchor system. Arthrosc Tech 2018 Aug 20;7:e927-e937.

16. Atoun E, Kane LT, Abboud JA. Arthroscopic, needlebased, transosseous rotator cuff repair. Arthrosc Tech 2020;9:e57-e63.

17. Sandow MJ, Schutz CR. Arthroscopic rotator cuff repair using a transosseous knotless anchor (ATOK). J Shoulder Elbow Surg 2020;29:527-533.

18. Sanders B. True transosseous hybrid rotator cuff repair. Arthrosc Tech 2019;8:e1013-e1018.

19. Hirakawa Y, Manaka T, Ito Y, et al. Intraoperative bone tunnel laceration affects integrity of arthroscopic transosseous rotator cuff repair with a tunneling device. Curr Orthop Pract 2019;30:336-342.

20. Black EM, Lin A, Srikumaran U, Jain N, Freehill MT. Arthroscopic transosseous rotator cuff repair: Technical note, outcomes, and complications. Orthopedics 2015;38: e352-e358.

21. Liu XN, Yang CJ, Lee GW, Kim SH, Yoon YH, Noh KC. Functional and radiographic outcomes after arthroscopic transosseous suture repair of medium sized rotator cuff tears. Arthroscopy 2018;34:50-57.

22. Chillemi C, Dei Giudici L, Mantovani M, Osimani M, Gumina S. Rotator cuff failure after surgery: An allarthroscopic transosseous approach. Musculoskelet Surg 2018;102:3-12.

23. Steinitz A, Buxbaumer P, Hackl M, Buess E. Arthroscopic transosseous anchorless rotator cuff repair using the X-Box technique. Arthrosc Tech 2019;8:ppe175-ppel81.

24. Constant CR, Gerber C, Emery RJH, Soibjerg JO, Gohlke F, Boileau P. A review of the Constant score: Modifications and guidelines for its use. J Shoulder Elbow Surg 2007;17:355-361.
25. Sugaya H, Maeda K, Matsuki K, Moriishi J. Functional and structural outcome after arthroscopic full-thickness rotator cuff repair: Single-row versus dual-row fixation. Arthroscopy 2005;21:1307-1316.

26. Harris JD, Brand JC, Cote MP, Faucett SC, Dhawan A. Research pearls: The significance of statistics and perils of pooling. Part 1: Clinical versus statistical significance. Arthroscopy 2017;33:1102-1112.

27. Randelli P, Stoppani CA, Zaolino C, Menon A, Randelli F, Cabitza P. Advantages of arthroscopic rotator cuff repair with a transosseous suture technique: A prospective randomized controlled trial. Am J Sports Med 2017;45:2000-2009.

28. Caldwell GL, Warner JJP, Miller MD, Boardman D, Towers J, Debski R. Strength of fixation with transosseous sutures in rotator cuff repair. J Bone Joint Surg Am 1997;79:1064-1068.

29. Koh JL, Szomor Z, Murrell GAC, Warren RF. Supplementation of rotator cuff repair with a bioresorbable scaffold. Am J sports Med 2002;30:410-413.

30. Tauber M, Hoffelner T, Penzkofer R, et al. Arthroscopic rotator cuff repair: A biomechanical comparison of the suture-bridge technique vs. a new transosseous technique using SutureButtons. Clin Biomech 2011 ; 26:910-916.

31. Salata MJ, Sherman SL, Lin EC, et al. Biomechanical evaluation of transosseous rotator cuff repair. Am J Sports Med 2012;42:283-290.

32. Galatz LM, Ball CM, Teefey SA, Middleton WD, Yamaguchi K. The outcome and repair integrity of completely arthroscopically repaired large and massive rotator cuff tears. J Bone Joint Surg Am 2004;86-A:21 9-224.

33. Choi S, Kim MK, Kim GM, Roh YH, Hwang IK, Kang H. Factors associated with clinical and structural outcomes after arthroscopic rotator cuff repair with a suture bridge technique in medium, large, and massive tears. J Shoulder Elbow Surg 2014;23:1675-1681.

34. Ladermann A, Denard PJ, Collin P. Massive rotator cuff tears: Definition and treatment. Int Orthop 2015;39: 2403-2414.

35. Alidousti A, Mirzaee F, Bahramian F, Zafarani Z, Mirzaei N, Aslani H. Repair of massive and irreparable rotator cuff tear using arthroscopic method. J Lasers Med Sci 2018;9:168-176.

36. Oh JH, Park MS, Rhee SM. Treatment strategy for irreparable rotator cuff tears. Clin Orthop Surg 2018;10: 119-134. 